Article

\title{
Short Barriers for Lowering Current-Density in Terahertz Quantum Cascade Lasers
}

\author{
Liang Gao ${ }^{1} \mathbb{D}$, John L. Reno ${ }^{2}$ and Sushil Kumar ${ }^{1, * \mathbb{D}}$ \\ 1 Department of Electrical and Computer Engineering, Lehigh University, Bethlehem, PA 18015, USA; \\ lig214@lehigh.edu \\ 2 Center for Integrated Nanotechnologies, Sandia National Laboratories, MS 1303, \\ Albuquerque, NM 87185-1303, USA; jlreno@sandia.gov \\ * Correspondence: sushil@lehigh.edu
}

Received: 3 December 2019; Accepted: 2 January 2020; Published: 8 January 2020

\begin{abstract}
Scattering due to interface-roughness (IR) and longitudinal-optical (LO) phonons are primary transport mechanisms in terahertz quantum-cascade lasers (QCLs). By choosing $\mathrm{GaAs} / \mathrm{Al}_{0.10} \mathrm{Ga}_{0.90}$ As heterostructures with short-barriers, the effect of IR scattering is mitigated, leading to low operating current-densities. A series of resonant-phonon terahertz QCLs developed over time, achieving some of the lowest threshold and peak current-densities among published terahertz QCLs with maximum operating temperatures above $100 \mathrm{~K}$. The best result is obtained for a three-well 3.1 THz QCL with threshold and peak current-densities of $134 \mathrm{~A} / \mathrm{cm}^{2}$ and $208 \mathrm{~A} / \mathrm{cm}^{2}$ respectively at $53 \mathrm{~K}$, and a maximum lasing temperature of $135 \mathrm{~K}$. Another three-well QCL designed for broadband bidirectional operation achieved lasing in a combined frequency range of 3.1-3.7 THz operating under both positive and negative polarities, with an operating current-density range of $167-322 \mathrm{~A} / \mathrm{cm}^{2}$ at $53 \mathrm{~K}$ and maximum lasing temperature of $141 \mathrm{~K}$ or $121 \mathrm{~K}$ depending on the polarity of the applied bias. By showing results from QCLs developed over a period of time, here we show conclusively that short-barrier terahertz QCLs are effective in achieving low current-density operation at the cost of a reduction in peak temperature performance.
\end{abstract}

Keywords: terahertz lasers; quantum cascade lasers; interface roughness scattering; LO-phonon scattering

\section{Introduction}

Significant progress has been made in operating temperature, output power, beam quality and spectral characteristics of terahertz ( $\mathrm{THz}$ ) quantum cascade lasers (QCLs) since their first demonstration in 2002 [1,2], which makes QCLs arguably the most promising sources of coherent terahertz radiation for diverse applications in terahertz spectroscopy, sensing, and imaging. Maximum operating temperatures in the range of 160-210 K have been realized for QCLs designed to emit in the frequency range of 2-4 THz [3-6] with recent demonstration of thermoelectrically cooled QCLs [6,7]. However, to achieve radiation in a good quality beam with acceptable amount of optical power, terahertz QCLs still need to be cooled to temperatures below $100 \mathrm{~K}$ [2]. There is an increasing use of compact liquid cryogen-free coolers such as Stirling coolers to operate such QCLs at 50-70 K [8,9]. Lowering of the electrical power consumption for terahertz QCLs is an important goal due to the limited cooling capacity of such miniature cryocoolers, which makes lowering of the operating current-densities of paramount importance while maintaining reasonably high maximum lasing temperatures.

The current-transport in the superlattice of a terahertz QCL is impacted by a complex interplay of various non-radiative scattering mechanisms that include LO-phonon scattering, IR scattering, and impurity scattering. The role of IR scattering as a dominant non-radiative mechanism, which reduces the lifetime of the upper laser subbands (and hence, the peak gain) and increases the operating 
current-densities of terahertz QCLs, has been studied theoretically [10,11]. Ref. [12] demonstrated experimental proof of IR scattering having noticeable impact on transport and lasing performance of terahertz QCLs by producing symmetrical lasers from materials, in which IR scattering depends on growth direction. The relative importance of other intersubband transport mechanisms such as inter-well resonant-tunneling between electrons subbands as well as scattering-assisted leakage of confined electrons into the energy continuum over the heterostructure barriers is poorly understood, which makes simultaneous optimization of high-temperature gain and low current-density operation in terahertz QCLs challenging.

The best performing terahertz QCLs in terms of output power and operating temperature implement different variations of LO-phonon scattering mediated depopulation mechanisms. Arguably, the simplest of such designs use a three-well $[3,5,13]$ repeat-period in the superlattice with a resonant-phonon depopulation scheme [14]. The three-well bandstructure is characterized by ease of design owing to relatively small number of superlattice parameters that need to be adjusted to realize the desired radiative frequency. However, such QCLs are typically characterized by large $\left(>500 \mathrm{~A} / \mathrm{cm}^{2}\right.$ ) operating current-densities due to strong parasitic current channels in the superlattice. Despite a large number of different types of terahertz QCLs that have been published, there only a few that simultaneously achieved a large $(>100 \mathrm{~K})$ maximum operating temperature and low $\left(\lesssim 300 \mathrm{~A} / \mathrm{cm}^{2}\right)$ operating (threshold and peak) current-densities [15-19]. In this paper, we show that shorter (and hence thicker) barriers could be utilized for such terahertz QCLs to mitigate the role of IR scattering, which leads to significant lowering of the threshold and peak current-densities, albeit in exchange of worsened maximum lasing temperatures. Experimental results from two different QCLs based on the three-well resonant-phonon design scheme are presented, both of which show low threshold and maximum current-densities. The best result is achieved for a $3.1 \mathrm{THz}$ QCL with a threshold and maximum current-density of $134 \mathrm{~A} / \mathrm{cm}^{2}$ and $208 \mathrm{~A} / \mathrm{cm}^{2}$ respectively when operating in pulsed mode at $53 \mathrm{~K}$, and a maximum operating temperature of $\sim 135 \mathrm{~K}$ at a current-density of $\sim 280 \mathrm{~A} / \mathrm{cm}^{2}$. These are significantly lower current-densities for any three-well terahertz QCL published in the literature with uniform barriers. The results are comparable in performance to a step-well terahertz QCL [18], which is more challenging to grow, and a four-well resonant-phonon terahertz QCL [17], which is typically more challenging to design and optimize.

\section{Role of IR Scattering in Three-Well QCLs with Short and Tall Barriers}

To study the role of IR scattering in resonant-phonon terahertz QCLs, we redesigned the three-well $3 \mathrm{THz}$ QCL structure in the $\mathrm{GaAs} / \mathrm{Al}_{0.15} \mathrm{Ga}_{0.85} \mathrm{As}$ material-system that has shown maximum operating temperatures in the range of 175-200 K [5,20]. The QCL was redesigned with shorter (and hence, thicker) $\mathrm{Al}_{0.10} \mathrm{Ga}_{0.90}$ As barriers. This modified design, named as RTRP3W198, maintains approximately similar injection/collection coupling strengths for resonant-tunneling and subband energy-separations compared to the reference design of Ref. [5]. However, the oscillator strength of the lasing transition was reduced from 0.56 to 0.42 to make the design more diagonal, which serves to suppress parasitic current channels [3]. To better understand how shorter barriers lead to lowering of the operating current-density, a simplified density-matrix transport model as reported in Ref. [21] is utilized to compute the current-density, and subband populations, and gain spectrum as a function of frequency in both the designs. Figure 1a shows the computed electron wavefunctions in a tight-binding formalism for bias corresponding to peak-gain in the typical three-well resonant-phonon QCL bandstructure that are used to compute the transport characteristics. The tight-binding wavefunctions are useful to model the inter-sub-module electron transport via resonant-tunneling. A somewhat arbitrary phenomenological dephasing time of $T_{2}^{*} \sim 0.25 \mathrm{ps}$ is used [3] that leads to current-densities that are similar to experimentally measured values. For simplicity, the electron temperature is kept the same as the lattice temperature for computation of subband lifetimes (which primarily affects LO-phonon scattering times). The intersubband transition rates for IR scattering are calculated as in Ref. [22] with an assumed mean height of roughness of $\Delta=0.4 \mathrm{~nm}$ and a correlation length of $\Lambda=7.5 \mathrm{~nm}$ [23] 
which are also phenomenological parameters. Alloy disorder scattering and impurity scattering that are relatively weaker are neglected in the model.
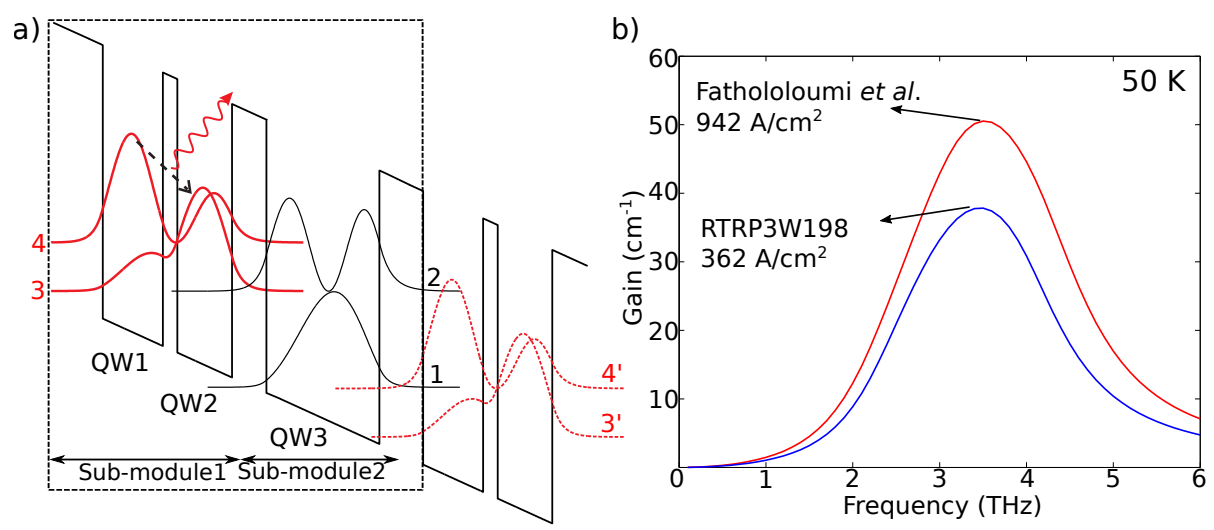

Figure 1. (a) Plot of magnitude-squared wavefunctions computed in a tight-binding formalism for a typical three-well resonant-phonon terahertz quantum cascade laser (QCL) design at the bias corresponding to peak-gain. A QCL module is divided into two sub-modules at barriers affecting transport of electrons via resonant-tunneling (RT). The radiative transition $4 \rightarrow 3$ occurs in the sub-module consisting of quantum-wells QW 1 and QW 2, whereas the injector and extraction subbands 2 and 1 respectively are localized in the sub-module comprising of quantum-well QW 3. (b) Theoretically calculated gain spectra using a simplified density-matrix transport model for the three-well resonant-phonon designs RTRP3W198 (with short Al-10\% barriers) and the design from Fathololoumi et al. [5] (with taller Al-15\% barriers) respectively, at the bias corresponding to peak-gain. In the model, the electron and lattice temperatures are set to $50 \mathrm{~K}$ and the inter-module electron transport occurs via RT whereas intra-module transport is modeled by electron-LO-phonon scattering and interface-roughness scattering.

Numerically computed gain spectra for both the designs for the peak-gain bias at a temperature of $50 \mathrm{~K}$ are shown in Figure 1b. Other details from the computation are summarized in Table 1. The calculated peak gain frequencies are higher than the lasing frequencies for both devices, the discrepancy is due to coherent resonant-tunneling assisted depopulation as discussed in [21]. The key result is a significant lowering of the current-density by $\sim 40 \%$ from $\sim 940 \mathrm{~A} / \mathrm{cm}^{2}$ in the tall-barrier design to $360 \mathrm{~A} / \mathrm{cm}^{2}$ in the short-barrier design. A reduction of peak-gain by $\sim 25 \%$ from $51 \mathrm{~cm}^{-1}$ to $38 \mathrm{~cm}^{-1}$ also occurs, which is primarily due to an almost similar fractional reduction in the radiative oscillator strength of the respective designs. It can therefore be concluded that lowering of current-density does not negatively impact gain, and hence, a high current-density is not a necessary requirement for high-gain. The lifetime of the upper radiative subband due to $4 \rightarrow 3$ IR scattering $\left(\tau_{\mathrm{IR}, 4 \rightarrow 3}\right)$ is $\sim 2.6$ times longer in the short-barrier QCL RTRP3W198 compared to the reference design. This is directly reflected in experimental results as shown subsequently, and is the primary benefit of using shorter and thicker barriers that suppress IR scattering. The density-matrix model predicts a relatively large $\left(\sim 31 \mathrm{~cm}^{-1}\right)$ gain even at high-temperatures for the short-barrier QCL as seen from the computation results at $150 \mathrm{~K}$ in Table 1 . It may be noted, however, that presumably increased thermal leakage into continuum at higher operating temperatures should lead to rapid degradation in gain with temperature, thereby lowering its the maximum lasing temperature considerably in such a design. This is indeed what is observed experimentally. 
Table 1. Key parameters related to the density-matrix transport model used for the three-well resonant-phonon designs RTRP3W198 (with short-barriers) and that by Fathololoumi et al. [5] (with taller barriers), computed for the bias corresponding to maximum gain. Electron temperatures in the subbands was assumed to be same as that of the lattice temperature $T$ (as indicated) for simplicity and a general lack of consensus in terahertz QCL literature about the relation between the two.

\begin{tabular}{|c|c|c|c|c|c|c|c|}
\hline Design & $\mathrm{T}(\mathrm{K})$ & $\tau_{\mathrm{LO}, 4 \rightarrow 3}$ & $\tau_{\mathrm{IR}, 4 \rightarrow 3}$ & $\tau_{\text {tot }, 4 \rightarrow 3}$ & $\tau_{2 \rightarrow 1}$ & $\mathrm{~J}\left(\mathrm{~A} / \mathrm{cm}^{2}\right)$ & Peak Gain $\left(\mathrm{cm}^{-1}\right)$ \\
\hline RTRP3W198 & 50 & $148 \mathrm{ps}$ & $5.9 \mathrm{ps}$ & $5.7 \mathrm{ps}$ & $0.56 \mathrm{ps}$ & 362 & 38 \\
\hline Fathololoumi et al. [5] & 50 & 66 ps & $2.3 \mathrm{ps}$ & $2.2 \mathrm{ps}$ & $0.21 \mathrm{ps}$ & 942 & 51 \\
\hline RTRP3W198 & 150 & $4.0 \mathrm{ps}$ & $7.3 \mathrm{ps}$ & $2.6 \mathrm{ps}$ & $0.28 \mathrm{ps}$ & 666 & 31 \\
\hline Fathololoumi et al. [5] & 150 & $2.3 \mathrm{ps}$ & $2.8 \mathrm{ps}$ & $1.3 \mathrm{ps}$ & $0.20 \mathrm{ps}$ & 1353 & 37 \\
\hline
\end{tabular}

\section{Experimental Results from Two Different QCLs}

Figure 2a shows conduction band-diagram of RTRP3W198 as designed, at peak-gain bias of $47 \mathrm{mV} /$ module $(9.2 \mathrm{kV} / \mathrm{cm})$ with the key design parameters. RTRP3W198 was grown by molecular beam epitaxy with 198 cascaded modules, with $\mathrm{n}=5 \times 10^{18} \mathrm{~cm}^{-3}$ contact layers grown above $(60 \mathrm{~nm}$ thick) and below ( $50 \mathrm{~nm}$ thick) the 10- $\mu \mathrm{m}$ thick active region. The exposed top contact layer adds extra loss and hence, for ease of fabrication, it is removed entirely. However, for negative polarity operation as shown in the following text, the contact layer must be kept under the metal for biasing. A $200 \mathrm{~nm} \mathrm{Al}_{0.55} \mathrm{Ga}_{0.45} \mathrm{As}$ etch-stop layer underlies the entire growth. Details regarding epitaxy of teraherz QCLs could be found in Refs [24,25]. A metal-metal waveguide was fabricated by a standard $\mathrm{Cu}-\mathrm{Cu}$ wafer bonding and processed using the method as shown in Ref. [26]. Ta/Cu/Au layers were deposited as both top (18/200/150 nm) and bottom $(18 / 200 / 150 \mathrm{~nm})$ contact to lower the waveguide losses [27]. Ridges were processed by wet-etching using 1:8:80:: $\mathrm{H}_{2} \mathrm{SO}_{4}: \mathrm{H}_{2} \mathrm{O}_{2}: \mathrm{H}_{2} \mathrm{O}$ etchant. The fabricated devices were cleaved and indium soldered on copper mount, wire bonded and mounted on the cold-stage of a Stirling cooler.
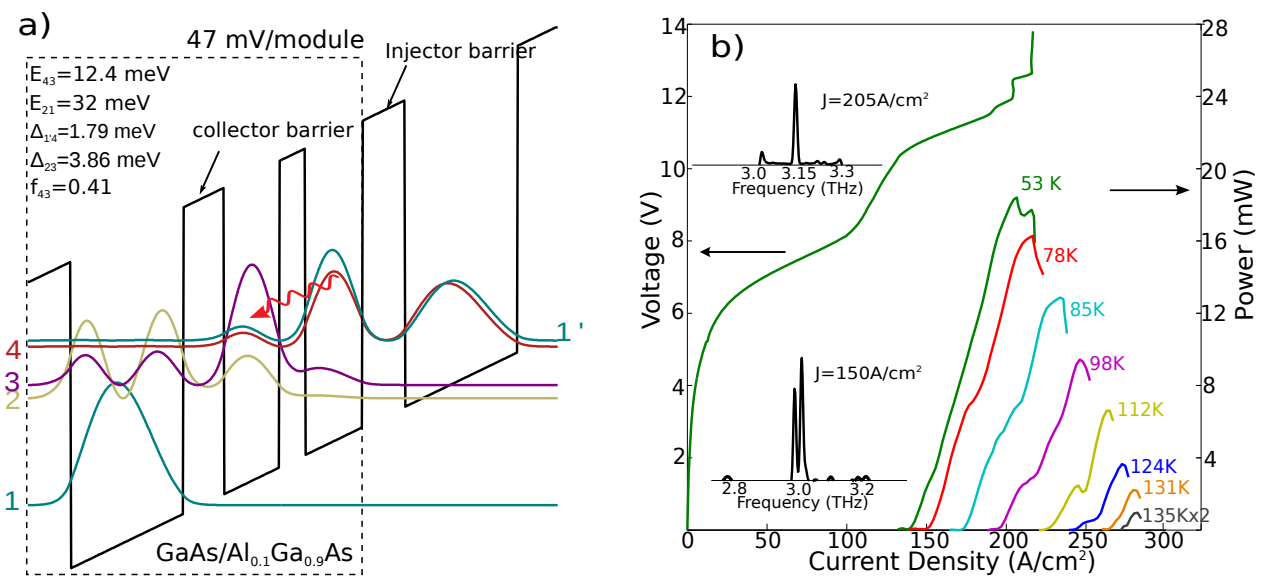

Figure 2. (a) One module conduction band diagram of the short-barrier three-well resonant-phonon QCL design named RTRP3W198 at the peak-gain bias. Starting from injector barrier, layer thicknesses in monolayers (ML) are (with barriers indicated in bold face) 23/31/14/30/22/61 where the center of the widest well is $n$-doped with sheet-density of $2.8 \times 10^{10} \mathrm{~cm}^{-2}$. The radiative transition is between $4 \rightarrow 3$ where $\mathrm{E}_{43}=12.4 \mathrm{meV}(\sim 3 \mathrm{THz})$ and the radiative oscillator strength $\mathrm{f}_{43}=0.41 . \Delta_{\mathrm{mn}}$ is the energy splitting between subbands $m$ and $n$ when they are aligned for optimal resonant-tunneling. $\Delta_{32}=$ $3.86 \mathrm{meV}$. (b) Experimental light-current-voltage $(L-I-V)$ characteristics from a $3.2 \mathrm{~mm} \times 120 \mu \mathrm{m}$ ridge laser with metal-metal cavity. A threshold current-density of $134 \mathrm{~A} / \mathrm{cm}^{2}$ and a maximum of $208 \mathrm{~A} / \mathrm{cm}^{2}$ was measured at $53 \mathrm{~K}$ in pulsed mode of operation with $300 \mathrm{~ns}$ pulses repeated at $100 \mathrm{kHz}$. Insets show the representative spectra of the QCL measured at $53 \mathrm{~K}$. The QCL emits in the frequency range of 3.0-3.2 THz.

Pulsed L-I-V characteristics and temperature performance of a representative RTRP3W198 QCL with its spectra are shown in Figure $2 \mathrm{~b}$. The QCL radiated at $\sim 3.1 \mathrm{THz}$ and the measured threshold and peak current-densities at $53 \mathrm{~K}$ heat-sink temperature were $134 \mathrm{~A} / \mathrm{cm}^{2}$ and $208 \mathrm{~A} / \mathrm{cm}^{2}$ respectively. 
The corresponding peak optical power as detected by the power meter was $19 \mathrm{~mW}$. Its maximum lasing temperature was $135 \mathrm{~K}$. In comparison to the QCLs in Refs. [5,20] a loss in the maximum lasing temperature by $\sim 40-65 \mathrm{~K}$ is compensated by a significant reduction (by a factor of $\sim 7$ ) in the operating current-densities, and hence, the electrical power dissipation. The latter is an important performance enhancement if the QCL was to be operated below $100 \mathrm{~K}$ in a miniature cryocooler, notwithstanding its lower maximum operating temperature. This result also indicates that while IR scattering plays an important role in electron transport at low-temperatures, it is not the primary mechanism limiting maximum operating temperatures of resonant-phonon terahertz QCLs.

One of the unique features of QCLs is that they could be designed to emit at two different frequencies independently when biased with positive and negative electrical polarities respectively. This is possible because of the unipolar carrier transport in intersubband superlattice. The gain bandwidth of terahertz QCLs based on resonant-phonon designs is typically in the range of $0.2-0.5 \mathrm{THz}$. So, bidirectional QCLs could double the gain bandwidth to potentially $\sim 1 \mathrm{THz}$ when properly designed for a monolithic QCL semiconductor chip. Terahertz QCLs with a broadband gain medium could play an important role in sensing and spectroscopy applications. There have been very few reports of bidirectional terahertz QCLs. We reported one bidirectional QCL design which showed broadband lasing in the frequency range of $3.1-3.7 \mathrm{THz}$ with maximum operating temperature of $147 \mathrm{~K}$ and $128 \mathrm{~K}$ respectively in opposing polarities [28]. That QCL was grown with a thinner $(\sim 8 \mu \mathrm{m})$ active region, which has higher waveguide losses for metallic cavities. Here, we present a regrowth of the same design, when grown to a $10 \mu \mathrm{m}$ thickness that is typical for terahertz QCLs based on metallic cavities. The design, named BIDR3W198, has same design parameters as [28]. Figure 3a shows the conduction band diagram of BIDR3W198 in forward-bias, and Figure 3b in reverse-bias respectively for the same superlattice structures. A key distinguishing feature for a bidirectional design is the use of barriers of same thickness for injection and extraction of electrons from radiative subband by resonant-tunneling. This is evident from the similar energy anticrossing parameters $\Delta_{1^{\prime} 4}$ and $\Delta_{32}$ in both polarities that primarily determine the strength of resonant-tunneling transport.

The bidirectional QCL is fabricated following the same process as RTRP3W198 QCL while the top contact layer is kept for negative bias. This increases cavity loss for negative operation and impacts (degrades) the maximum operating temperature. Pulsed $L-I-V$ characteristics and temperature performance of a representative BIDR3W198 QCL in opposing polarities are shown in Figure 2c,d respectively. The threshold current-densities in opposing polarities are $167 \mathrm{~A} / \mathrm{cm}^{2}$ and $217 \mathrm{~A} / \mathrm{cm}^{2}$ respectively at $53 \mathrm{~K}$, which is lower than the values in Ref. [28] owing to lower waveguide losses and also possibly due to growth including possibly doping variations. Note that the maximum lasing temperatures of BIDR3W198 are lower than that in Ref. [28] in both polarities. We note the $I-V$ s near peak bias of BIDR3W198 in both polarities are very rough which may indicate slight growth problems. Nevertheless, the experimental results show that bidirectional terahertz QCLs based on short-barriers are effective in providing broadband gain from the same superlattice structure with low operating current-densities and reasonably high maximum operating temperatures. 


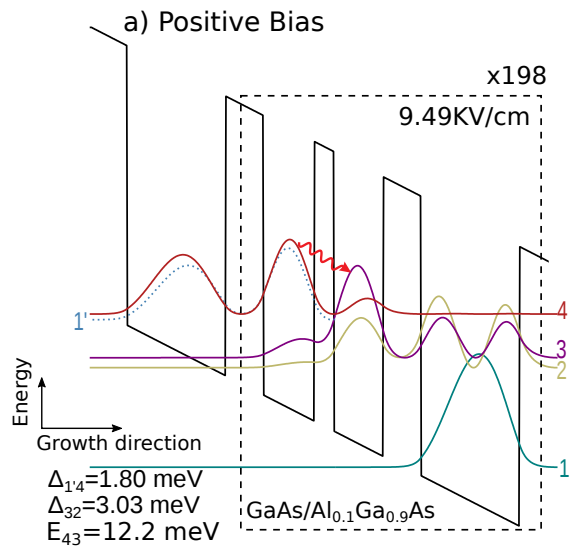

b) Negative Bias
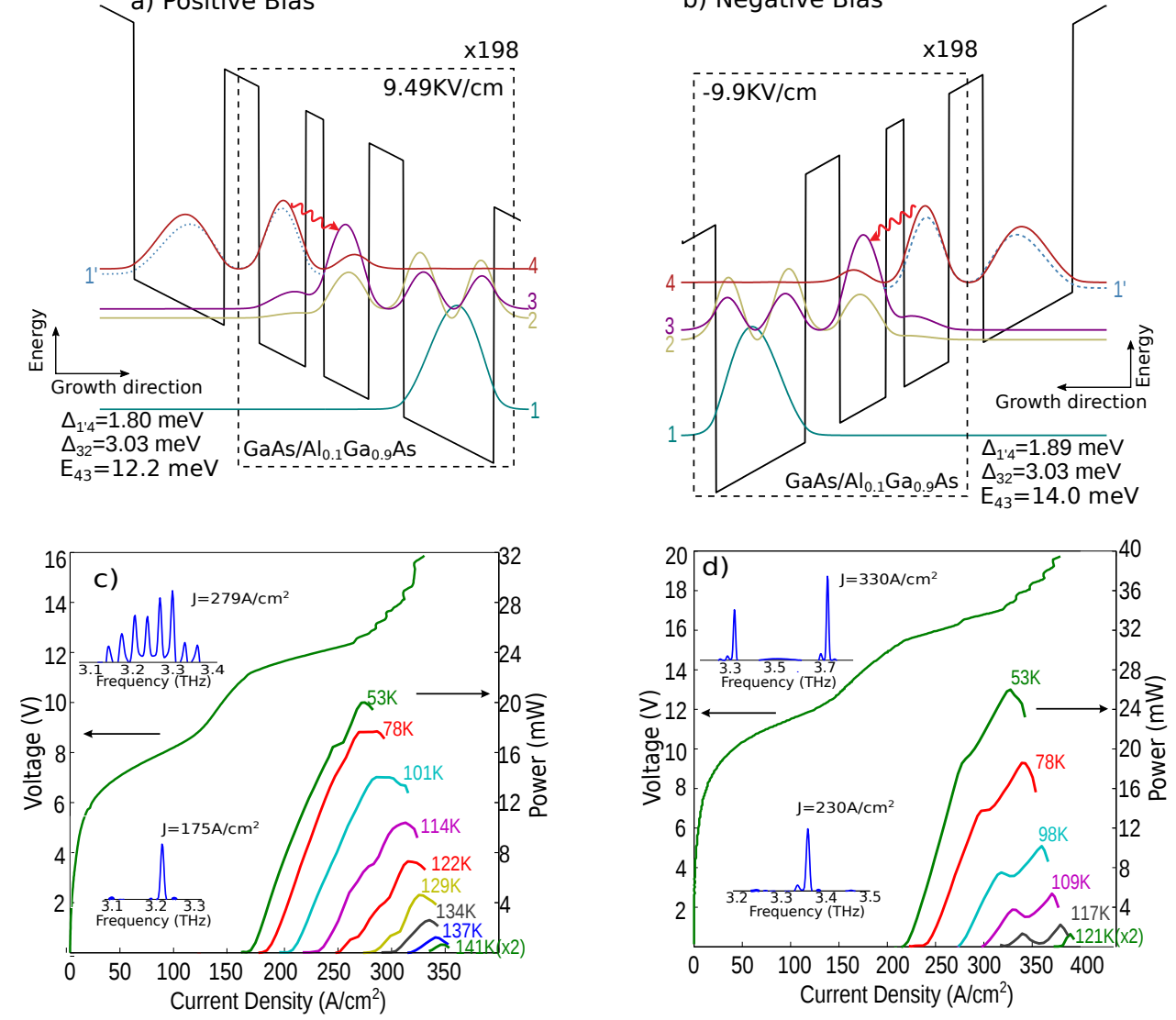

Figure 3. (a) and (b) Conduction band diagrams for a bidirectional three-well resonant-phonon terahertz QCL design named BIDR3W198 at peak-gain bias corresponding to positive and negative polarity operation respectively. In contrast to RTRP3W198 of Figure 2, the bidirectional design BIDR3W198 is characterized by injection and extraction barriers of same thickness. Starting from injector barrier, layer thicknesses in monolayers (ML) (where barriers are indicated in bold face) are

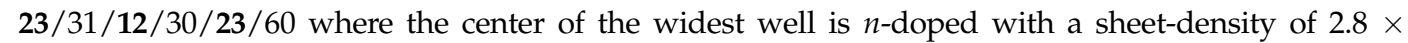
$10^{10} \mathrm{~cm}^{-2}$. Key design parameters are indicated alongside the band diagrams. (c) and (d) Experimental light-current-voltage $(L-I-V)$ characteristics from a $1.6 \mathrm{~mm} \times 120 \mu \mathrm{m}$ ridge laser with metal-metal cavities biased under positive and negative polarity respectively. The threshold current-densities at positive and negative polarity bias are $167 \mathrm{~A} / \mathrm{cm}^{2}$ and $217 \mathrm{~A} / \mathrm{cm}^{2}$ respectively at $53 \mathrm{~K}$, and the corresponding maximum operating temperatures are $141 \mathrm{~K}$ and $121 \mathrm{~K}$ in pulsed mode of operation. Pulsed $L-I-V$ measurement was done with $300 \mathrm{~ns}$ pulses repeated at $100 \mathrm{kHz}$. The QCL radiates in the frequency range 3.1-3.4 THz under positive polarity and 3.3-3.7 THz under negative polarity bias respectively. Representative spectra measured at $53 \mathrm{~K}$ are shown the insets of the corresponding figures.

\section{Summary}

Table 2 shows a summary of the performances of three different three-well resonant-phonon terahertz QCL structures based on the GaAs $/ \mathrm{Al}_{0.10} \mathrm{Ga}_{0.90}$ As material system with short-barriers compared to the conventional $\mathrm{Al}_{0.15} \mathrm{Ga}_{0.85}$ As barriers used in literature. The RTRP3W197 QCL was grown several years ago and was briefly reported in Ref. [29]. The results presented in this manuscript with new designs that are grown at different time-periods now conclusively show the benefit of using short-barriers to suppress IR scattering in resonant-phonon terahertz QCLs, which, in conjunction with the use of a diagonal radiative transition [3], can lead to a significant lowering of operational current-density in such QCLs. While this benefit comes at the expense of lower maximum operating temperatures, the smaller electrical dissipation in the QCLs could be a significant advantage when such QCLs are operated below $100 \mathrm{~K}$ in miniature cryocoolers that have a limited cooling capacity. In the future, two QCLs based on short barriers (Al-10\%) and tall barriers (Al-15\%) with very similar design 
parameters, such as lasing frequency, oscillator strength, and subband coupling could be designed, fabricated and tested in order to give a direct conclusion about the impact of IR scattering on the performance of terahertz QCLs.

Table 2. Performance summary of various three-well resonant-phonon QCLs based on $\mathrm{GaAs} / \mathrm{Al}_{0.10} \mathrm{Ga}_{0.90} \mathrm{As}$ superlattices. The design RTRP3W197 was developed and grown several years ago and was briefly reported in Ref. [29]. It differs from RTRP3W198 of Figure 2 with a slightly thinner extraction barrier (by 1 ML).

\begin{tabular}{ccccccc}
\hline Design Name & Wafer No. & Frequency & Jth $\left(\mathbf{A} / \mathbf{c m}^{2}\right)$ & Jmax $\left(\mathbf{A} / \mathbf{c m}^{2}\right)$ & Tmax & Doping Density \\
\hline RTRP3W197 & VB0464 & $2.7 \mathrm{THz}$ & $240 @ 77 \mathrm{~K}$ & $450 @ 77 \mathrm{~K}$ & $157 \mathrm{~K}$ & $2.8 \times 10^{10} \mathrm{~cm}^{-2}$ \\
RTRP3W198 & VB0890 & $3.1 \mathrm{THz}$ & $134 @ 53 \mathrm{~K}$ & $208 @ 53 \mathrm{~K}$ & $135 \mathrm{~K}$ & $2.8 \times 10^{10} \mathrm{~cm}^{-2}$ \\
BIDR3W198 (pos. bias) & VB0891 & $3.2 \mathrm{THz}$ & $167 @ 53 \mathrm{~K}$ & $270 @ 53 \mathrm{~K}$ & $141 \mathrm{~K}$ & $2.8 \times 10^{10} \mathrm{~cm}^{-2}$ \\
BIDR3W198 (neg. bias) & VB0891 & $3.5 \mathrm{THz}$ & $217 @ 53 \mathrm{~K}$ & $322 @ 53 \mathrm{~K}$ & $121 \mathrm{~K}$ & $2.8 \times 10^{10} \mathrm{~cm}^{-2}$ \\
\hline
\end{tabular}

Author Contributions: L.G. and S.K. designed the quantum cascade lasers (QCLs). L.G. performed fabrication and did experimental characterization. J.L.R. was responsible for growth of the QCLs using molecular beam epitaxy. S.K. supervised the project. All authors have read and agreed to the published version of the manuscript.

Funding: This work is funded by the U.S. National Science Foundation under grants ECCS 1609168 and ECCS 1351142.

Acknowledgments: The semiconductor lasers were fabricated at nanofabrication facility of the Center for Photonics and Nanoelectronics at Lehigh University. This work was performed, in part, at the Center for Integrated Nanotechnologies, an Office of Science User Facility operated for the U.S. Department of Energy (DOE) Office of Science. Sandia National Laboratories is a multimission laboratory managed and operated by National Technology \& Engineering Solutions of Sandia, LLC, a wholly owned subsidiary of Honeywell International, Inc., for the U.S. DOE's National Nuclear Security Administration under contract DE-NA-0003525. The views expressed in the article do not necessarily represent the views of the U.S. DOE or the United States Government.

Conflicts of Interest: The authors declare no conflict of interest.

\section{References}

1. Köhler, R.; Tredicucci, A.; Beltram, F.; Beere, H.E.; Linfield, E.H.; Davies, A.G.; Ritchie, D.A.; Iotti, R.C.; Rossi, F. Terahertz semiconductor-heterostructure laser. Nature 2002, 417, 156-159. [CrossRef] [PubMed]

2. Zeng, Y.; Qiang, B.; Wang, Q.J. Photonic Engineering Technology for the Development of Terahertz Quantum Cascade Lasers. Adv. Opt. Mater. 2019. [CrossRef]

3. Kumar, S.; Hu, Q.; Reno, J.L. $186 \mathrm{~K}$ operation of terahertz quantum-cascade lasers based on a diagonal design. Appl. Phys. Lett. 2009, 94, 131105. [CrossRef]

4. Kumar, S.; Chan, C.W.I.; Hu, Q.; Reno, J.L. A $1.8 \mathrm{THz}$ quantum-cascade laser operating significantly above the temperature of $\hbar \omega / k_{\mathrm{B}}$. Nat. Phys. 2011, 7, 166. [CrossRef]

5. Fathololoumi, S.; Dupont, E.; Chan, C.W.I.; Wasilewski, Z.R.; Laframboise, S.R.; Ban, D.; Mátyás, A.; Jirauschek, C.; Hu, Q.; Liu, H.C. Terahertz quantum cascade lasers operating up to $\sim 200 \mathrm{~K}$ with optimized oscillator strength and improved injection tunneling. Opt. Express 2012, 20, 3866. [CrossRef]

6. Bosco, L.; Franckié, M.; Scalari, G.; Beck, M.; Wacker, A.; Faist, J. Thermoelectrically cooled THz quantum cascade laser operating up to $210 \mathrm{~K}$. Appl. Phys. Lett. 2019, 115, 010601. [CrossRef]

7. Kainz, M.A.; Semtsiv, M.P.; Tsianos, G.; Kurlov, S.; Masselink, W.T.; Schönhuber, S.; Detz, H.; Schrenk, W.; Unterrainer, K.; Strasser, G.; et al. Thermoelectric-cooled terahertz quantum cascade lasers. Opt. Express 2019, 27, 20688. [CrossRef]

8. Richter, H.; Greiner-Bär, M.; Pavlov, S.G.; Semenov, A.D.; Wienold, M.; Schrottke, L.; Giehler, M.; Hey, R.; Grahn, H.T.; Hübers, H.W. A compact, continuous-wave terahertz source based on a quantum-cascade laser and a miniature cryocooler. Opt. Express 2010, 18, 10177. [CrossRef]

9. Richter, H.; Wienold, M.; Schrottke, L.; Biermann, K.; Grahn, H.T.; Hübers, H.W. 4.7-THz local oscillator for the GREAT heterodyne spectrometer on SOFIA. IEEE Trans. Terahertz Sci. Technol. 2015, 5, 539. [CrossRef]

10. Franckié, M.; Winge, D.O.; Wolf, J.; Liverini, V.; Dupont, E.; Trinité, V.; Faist, J.; Wacker, A. Impact of interface roughness distributions on the operation of quantum cascade lasers. Opt. Express 2015, 23, 5201-5212. [CrossRef] 
11. Krivas, K.; Winge, D.; Franckié, M.; Wacker, A. Influence of interface roughness in quantum cascade lasers. J. Appl. Phys. 2015, 118, 114501. [CrossRef]

12. Deutsch, C.; Detz, H.; Zederbauer, T.; Andrews, A.M.; Klang, P.; Kubis, T.; Klimeck, G.; Schuster, M.E.; Schrenk, W.; Strasser, G.; et al. Probing scattering mechanisms with symmetric quantum cascade lasers. Opt. Express 2013, 21, 7209. [CrossRef] [PubMed]

13. Luo, H.; Laframboise, S.R.; Wasilewski, Z.R.; Aers, G.C.; Liu, H.C.; Cao, J.C. Terahertz quantum-cascade lasers based on a three-well active module. Appl. Phys. Lett. 2007, 90, 041112. [CrossRef]

14. Williams, B.S.; Callebaut, H.; Kumar, S.; Hu, Q.; Reno, J.L. 3.4-THz quantum cascade laser based on longitudinal-optical-phonon scattering for depopulation. Appl. Phys. Lett. 2003, 82, 1015-1017. [CrossRef]

15. Scalari, G.; Hoyler, N.; Giovannini, M.; Faist, J. Terahertz bound-to-continuum quantum-cascade lasers based on optical-phonon scattering extraction. Appl. Phys. Lett. 2005, 86, 181101. [CrossRef]

16. Kumar, S.; Williams, B.S.; Hu, Q.; Reno, J.L. $1.9 \mathrm{THz}$ quantum-cascade lasers with one-well injector. Appl. Phys. Lett. 2006, 88, 121123. [CrossRef]

17. Benz, A.; Fasching, G.; Andrews, A.M.; Martl, M.; Unterrainer, K.; Roch, T.; Schrenk, W.; Golka, S.; Strasser, G. Influence of doping on the performance of terahertz quantum-cascade lasers. Appl. Phys. Lett. 2007, 90, 101107. [CrossRef]

18. Scalari, G.; Amanti, M.I.; Fischer, M.; Terazzi, R.; Walther, C.; Beck, M.; Faist, J. Step well quantum cascade laser emitting at 3 THz. Appl. Phys. Lett. 2009, 94, 041114. [CrossRef]

19. Amanti, M.I.; Scalari, G.; Terazzi, R.; Fischer, M.; Beck, M.; Faist, J.; Rudra, A.; Gallo, P.; Kapon, E. Bound-to-continuum terahertz quantum cascade laser with a single-quantum-well phonon extraction/injection stage. New J. Phys. 2009, 11, 125022. [CrossRef]

20. Chan, C.W.I.; Hu, Q.; Reno, J.L. Tall-barrier terahertz quantum cascade lasers. Appl. Phys. Lett. 2013, $103,151117$. [CrossRef]

21. Kumar, $\mathrm{S}$; $\mathrm{Hu}, \mathrm{Q}$. Coherence of resonant-tunneling transport in terahertz quantum-cascade lasers. Phys. Rev. B 2009, 80, 245316. [CrossRef]

22. Sakaki, H.; Noda, T.; Hirakawa, K.; Tanaka, M.; Matsusue, T. Interface roughness scattering in GaAs/AlAs quantum wells. Appl. Phys. Lett. 1987, 51, 1934. [CrossRef]

23. Walther, C.; Fischer, M.; Scalari, G.; Terazzi, R.; Hoyler, N.; Faist, J. Quantum cascade lasers operating from 1.2 to 1.6 THz. Appl. Phys. Lett. 2007, 91, 131122. [CrossRef]

24. Beere, H.E.; Fowler, J.C.; Alton, J.; Linfield, E.H.; Ritchie, D.A.; Köhler, R.; Tredicucci, A.; Scalari, G.; Ajili, L.; Faist, J.; et al. MBE growth of terahertz quantum cascade lasers. J. Cryst. Growth 2005, 278, 756. [CrossRef]

25. Li, L.H.; Zhu, J.X.; Chen, L.; Davies, A.G.; Linfield, E.H. The MBE growth and optimization of high performance terahertz frequency quantum cascade lasers. Opt. Express 2015, 23, 2720. [CrossRef]

26. Williams, S.B.; Kumar, S.; Hu, Q.; Reno, J.L. Operation of terahertz quantum-cascade lasers at $164 \mathrm{~K}$ in pulsed mode and at $117 \mathrm{~K}$ in continuous-wave mode. Opt. Express 2005, 13, 3331. [CrossRef]

27. Belkin, A.M.; Fan, A.J.; Hormoz, S.; Capasso, F.; Khanna, S.; Lachab, M.; Davies, A.G.; Linfield, H.E. Terahertz quantum cascade lasers with copper metal-metal waveguides operating up to $178 \mathrm{~K}$. Opt. Express 2008, 16, 3242. [CrossRef]

28. Khanal, S.; Gao, L.; Zhao, L.; Reno, J.L.; Kumar, S. High-temperature operation of broadband bidirectional terahertz quantum-cascade lasers. Sci. Rep. 2016, 6, 32978. [CrossRef]

29. Khanal, S.; Zhao, L.; Reno, J.L.; Kumar, S. Temperature performance of terahertz quantum-cascade lasers with resonant-phonon active-regions. J. Opt. 2014, 16, 094001. [CrossRef]

(c) 2020 by the authors. Licensee MDPI, Basel, Switzerland. This article is an open access article distributed under the terms and conditions of the Creative Commons Attribution (CC BY) license (http:/ / creativecommons.org/licenses/by/4.0/). 